\title{
Outcome of Use of Barbed Sutures in Augmentation Mastopexy: A Case Series
}

\author{
WAEL NAEEM THABET AZIZ, M.D.* and AMR ABD EL-MONIEM EL-NAGGARY, M.D.** \\ The Department of General Surgery, Faculty of Medicine, Cairo University* and Department of Plastic Surgery, \\ Faculty of Medicine, Fayoum University**
}

\begin{abstract}
Widespread adoption of barbed suture usage in aesthetic procedures has been noticeable in the recent years. The use of barbed sutures in breast lift procedures during augmentation mastopexy is not new but needs more support in the literature. In this case series, we describe 15 surgeries of such with minimal complication rates and high satisfaction rates among surgeons and patients; this supports the usage of such an adjunct in augmentation mastopexy and endorses their safety.
\end{abstract}

Key Words: Barbed sutures - Breast lift - Breast ptosis Mastopexy.

\section{INTRODUCTION}

Barbed sutures are synthesized from monofilament materials. Although different technologies have been used to produce barbed sutures, the mechanism of action is the same for all types and involves hooking the tissues onto barbs of the thread so that they subsequently become encased in fibrous tissues, initiating a biologic response $[1,2]$.

There are many uses of barbed sutures in plastic surgery which became widely adopted in the recent years. Barbed suture devices were first used for minimally invasive facial rejuvenation techniques $[\mathbf{1 , 3 ]}$. Nowadays, more use of this technology is directed towards body contouring procedures like lifting significant skin redundancies and breast ptosis related to weight loss in order to improve operative outcome and in closure of large skin wounds $[4,5]$. The advantage of this technology of tissue closure is the speed and easy placement. Another advantage of barbed sutures is that the deeper suture layer is not often required and thus less operative time is required. In addition, lesser complications are associated with barbed sutures than with more conventional suture material, in- cluding extrusion and infection [2]. Finally, tissue tension may be uniformly distributed along the wound, and the hooking nature of the suture prevents tissue sliding with more than 20 points of fixation per square inch [6]. Some authors even go further to suggest that the final scar outcome is improved from a clinical perspective due to less tissue-related ischemia and less suture extrusion [3].

In the recent years, augmentation mastopexy with the aid of barbed sutures has gained both positive and negative attention. Yet, more studies are needed to establish the procedure's benefits and the risks. Combining the use of barbed sutures within breast mastopexy procedures does not, however, seem to increase risks in properly selected patients [7].

In this case series, 15 cases with Regnault's grade II or higher breast ptosis underwent single stage augmentation mastopexy using the unidirectional barbed 2-0 suture, and the post-operative results were assessed by both the operating surgeons and by the patients who answered self-satisfaction questionnaire.

\section{PATIENTS AND METHODS}

This study was conducted on 15 women who presented with breast ptosis and mammary hypotrophy, and required augmentation mastopexy at the same surgical time. Preoperative data recorded for each patient included age, body mass index (BMI), smoking status, co-morbidities, and the type of planned mastopexy (crescent, circumareolar, vertical, or inverted-T). Of the 15 cases, 3 cases had recurrent ptosis after previous augmentation mastopexy surgery done more than 3 years before. 
Inclusion criteria were medically fit cases without contraindications to anesthesia. Exclusion criteria included medically unfit cases, cases with unrealistic expectations, and those who did not accept the possibility of revision surgery.

A written informed consent was taken from each case with pre-operative digital photographs. Preoperative markings rely on the nipple areola complex (NAC) to suprasternal notch distance and nipple to the inframammary fold (IMF) distance.

Every patient who underwent the procedure received standardized care, including general inhalation anesthesia, perioperative antibiotics and application of lower-extremity compression.

\section{Surgical technique:}

Preoperative marking was done for all patients in standing position. Four areas were marked: The IMF, the midclavicular line, the suprasternal notch, and the new NAC in its proposed position. Patients were in supine position with 30 degrees elevation of the upper part of the table with arms abducted 90 degrees during the procedure.

All cases in this study were with NAC ptosis $3 \mathrm{~cm}$ or more below the IMF so vertical lift pattern was done. Local tissue infiltration with $40 \mathrm{ml}$ of mixture of saline $0.9 \%$ and adrenaline 1: 200,000 along the markings of incisions was carried out. By number 15 blade the vertical limb skin incision from the lower border of the areola down to point $2-3 \mathrm{~cm}$ above the IMF was done, then by electro cautery cutting mode the breast tissue was dissected down to the pectoral fascia which was elevated with the mammary gland to create a subfascial pocket that extended from $1 \mathrm{~cm}$ parasternal side to the anterior axillary line. A previously determined sizer was inserted in the pocket to accurately choose the suitable implant for each case, then by using a skin stapler, the pillars were approximated to take a primary idea about the projection and symmetry of both breasts. The sizers were removed then the implants were inserted after washing them with saline antibiotic (Gentamycin) solution.

NAC was repositioned to the new correct distance after excess skin depithelialization. Pillars were approximated by barbed sutures, $2 / 0$ size, $5 / 8$ rounded needle, unidirectional, starting from the medial pillar to the pectoralis muscle then to the lateral pillar. Three stiches were taken for each side.

No drains were needed for the primary (12) cases, but for the secondary (3) cases capsulectomies were done, so we preferred to put one suction drain size $14 \mathrm{~F}$ in each side to come out from the midaxillary point at the level of the IMF to avoid any possible seroma collection.

A second layer of continuous sutures was inserted between the pillars through the glandular tissue by barbed suture 3/0 unidirectional half circle rounded tip needle above the deeper sutures. The periareolar pattern was closed by bidirectional absorbable suture $3 / 0$ sutures run 2 times circumareolar to properly approximate the wound edges in this area. The skin closure was done by $3 / 0$ monofilimintous absorbable cutting tip needle. The appropriate pressure garment was applied for each patient.

\section{Post-operative:}

Close observation of vital signs and drains collection was done postoperatively. On discharge, the patients were instructed to keep wearing the pressure garment for 4 weeks and received the post-operative medications and follow-up appointments.

\section{Statistical analysis:}

All data were collected, tabulated and statistically analyzed using SPSS 19.0 for windows (SPSS Inc., Chicago, IL, USA). Quantitative data were expressed as mean \& standard deviation $(X \pm S D)$. Qualitative data were expressed as number and percentage (No. \& \%).

\section{RESULTS}

The patients' age ranged between $29-46$ years, the mean age was 37.6 years. As regards the assessment of the degree of ptosis, we used the distance between the suprasternal notch to nipple (SSN-N). This distance ranged between 26 to $33 \mathrm{~cm}$ with a mean of $28.9 \mathrm{~cm}$ and the NAC position was $3 \mathrm{~cm}$ or below in relation to the IMF. All patients were subjected to augmentation with vertical mastopexy technique using textured round high profile gel filled implants (Sebbin) brand which was inserted beneath the pectoral fascia in 7 cases and the 3 secondary cases was inserted sub (glandular/ submuscular) as none of these cases had capsular contracture.

The mean operative time was 93 minutes with a range of 85-102 minutes. The post-operative complications were stich sinus in 2 cases which were treated conservatively by dressing. Any postoperative edema resolved spontaneously.

Every patient (at 6 months postoperatively) was asked to answer a patient satisfaction questionnaire and to give a score from 1 (very disap- 
pointed) to 10 (very pleased) regarding each item of the following: Breast size, breast shape, breast symmetry, scars. The overall satisfaction rate was $86.7 \%$ among patients. The highest rates were seen for the breast shape responses, while the lowest were for the breast symmetry. Responses from 4 blinded surgeons were obtained, and a satisfied response was found in $72.5 \%$ of instances.

The difference between the mean satisfaction rates in the primary and secondary groups was not statistically significant for the surgeons' satisfaction $(p=0.674)$ nor for the patients' satisfaction ( $p=$ $0.741)$. There wasn't a statistically significant difference in outcome satisfaction between the complicated and non-complicated cases, neither for the surgeons $(p=0.423)$ nor for the patients $(p=0.391)$.

A sample of the patients' preoperative and postoperative appearance can be seen in Figs. (1-3).
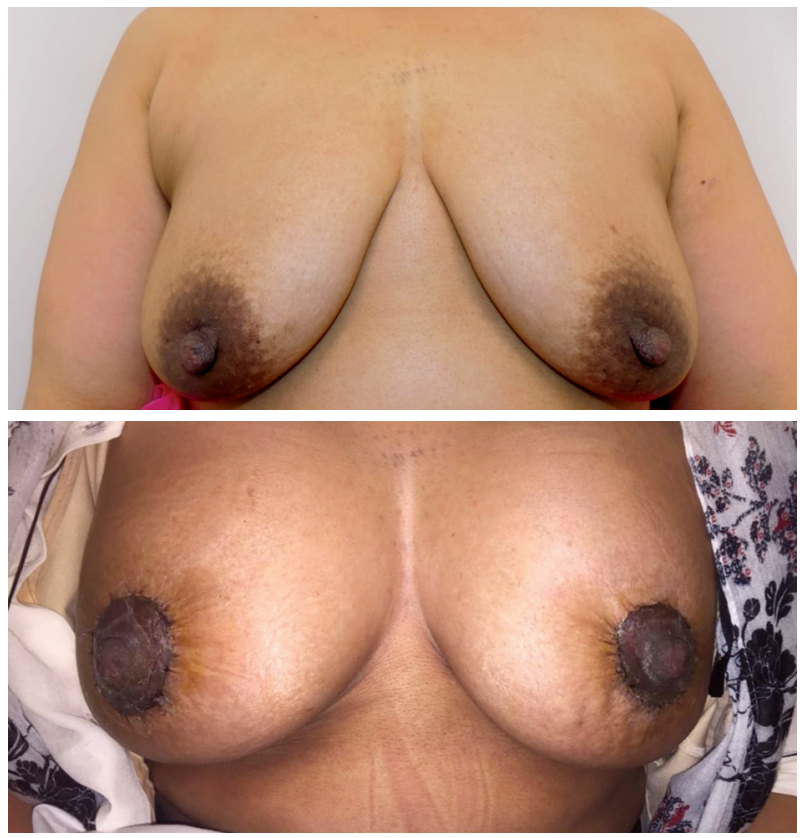

Fig. (1): Before (Upper panel) and After (Lower panel) images of Sample Case 1 in our study.
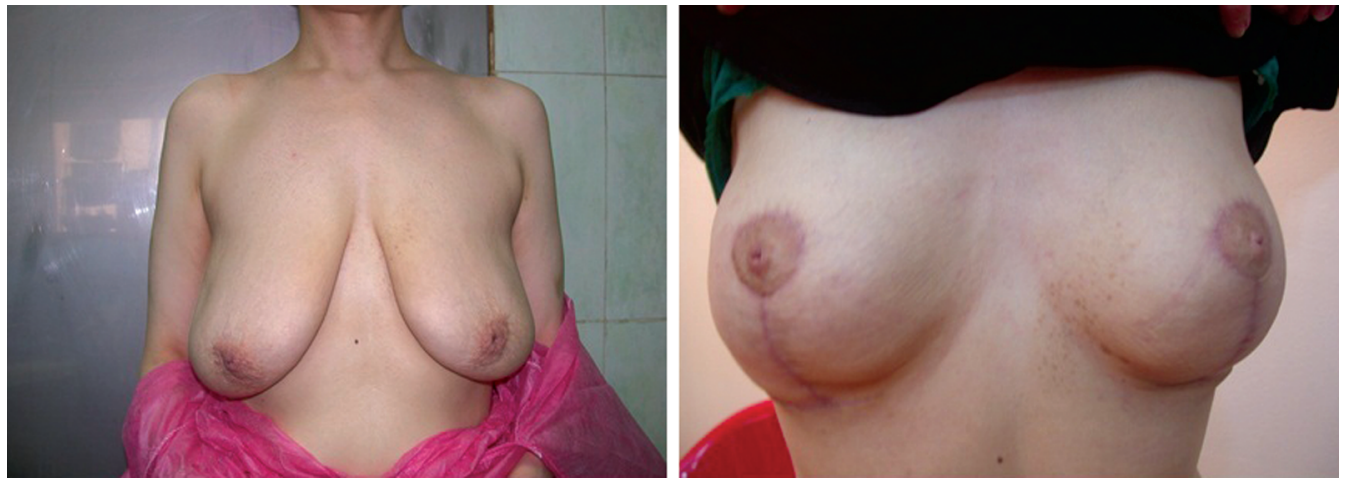

Fig. (2): Before (Left panel) and After (Right panel) images of Sample Case 2 in our study.

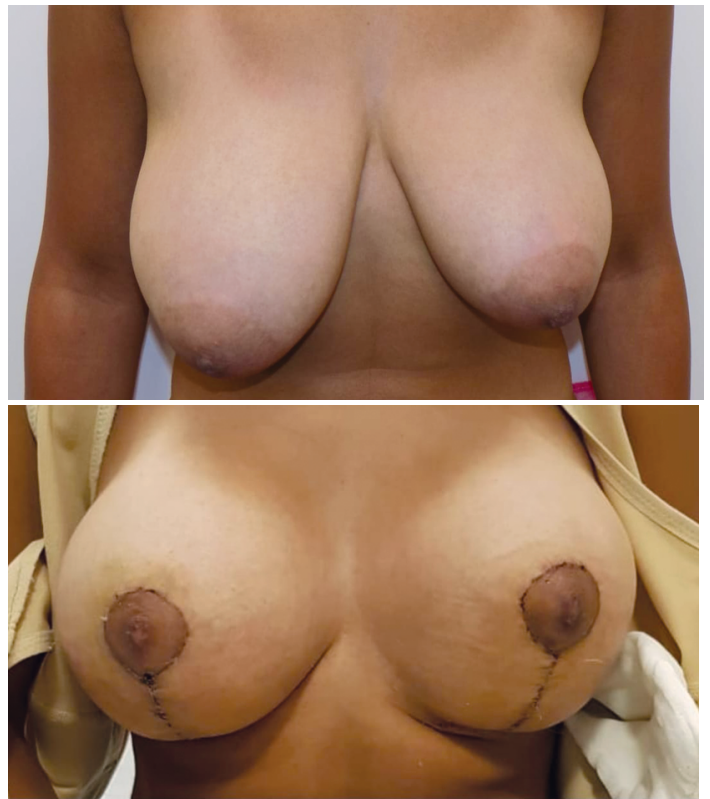

Fig. (3): Before (Upper panel) and After (Lower panel) images of Sample Case 3 in our study.

\section{DISCUSSION}

The presence of ptosis and mammary hypoplasia in the same patient is a common problem. The incidence of this issue has increased lately due to increased concern of women about weight loss which leads to breast ptosis and loss of volume [8]. There is no universal protocol on how it should be treated, or whether simultaneous surgical treatment is recommended.

Chanllenges of the combined augmentation mastopexy procedure include technical challenges and unpredictable results. However, satisfactory results with one stage augmentation mastopexy with acceptable aesthetic results were recently described [9]. Spear [10] has noted the greater likelihood of "major disasters" with the 1-stage procedure, including nipple loss and skin flap necrosis. However, we have not encountered these serious complications in our own experience. 
We chose subpectoral fascia plane of augmentation and not the dual plane placement to get the advantage of projection and avoid the motion artifact and possible lateralization of the implants associated with dual plane, and relied on the barbed sutures to hold the pillars and provide adequate support of the implant. This choice rationale was similar to Dancey and colleagues in their study [11].

Stevens and colleagues [12] demonstrated that the risks of one stage procedure are not more significant than those of two procedures performed separately. Therefore, they concluded that one stage augmentation mastopexy can be performed safely and with no greater risks than 2-stage procedures, which involve repeated surgery and anesthesia.

Ryan and colleagues [5] used barbed sutures in wound closure of different aesthetic surgery procedures and concluded that application of barbed sutures increased speed and efficiency of closures through smaller access incisions. This is in line with our data, where we recorded lower mean surgical durations than conventional approaches.

Hammond [3] reviewed that barbed sutures offer obvious benefits, including even distribution of tension along the wound, ease and accuracy of suture placement, elimination of the "third hand" during wound closure, avoidance of knots, shorter operative times, and the ability to provide fineline and inconspicuous scars. So the use of this technology is recommended for enhancing the surgical outcome for many plastic surgery patients.

In their retrospective study of 900 body contouring surgeries, Cannon [13] found that the use of 2 layers barbed sutures closure provided statistically significantly lower rates of wound-healing complications as compared with prior experience with traditional running braided absorbable sutures, more rapid closure, improved security of closure, and increased surgeon satisfaction with the process and wound-healing results. Our case series confirms such findings.

In conclusion, we found that use of barbed absorbable sutures a as support system for breast tissue in augmentation mastopexy is reliable and provides long lasting satisfying outcome for both patients and surgeons with no added risk of complications than other routine augmentation mastopexy techniques.

\section{REFERENCES}

1- Matarasso A. and Ruff G.L.: The History of Barbed Sutures. Aesthetic Surg J. Sep., 1; 33 (3 Supplement): 12S-16S, 2013.

2- Delorenzi C.: Barbed sutures: Rationale and technique. Aesthetic. Surg. J. Mar., 26 (2): 223-9, 2006.

3- Matarasso A. and Hammond D.C.: Barbed Sutures in Plastic Surgery. Aesthetic. Surg. J. Sep., 1; 33 (3 Supplement):32S-39S, 2013.

4- Greenberg J.A. and Goldman R.H.: Barbed suture: A review of the technology and clinical uses in obstetrics and gynecology. Rev. Obstet. Gynecol., 6 (3-4): 107-15, 2013.

5- Mitchell R.T.M. and Bengtson B.P.: Clinical Applications of Barbed Suture in Aesthetic Breast Surgery. Clin. Plast. Surg. Oct., 42 (4): 595-604, 2015.

6- Matarasso A. and Moya A.P.: Barbed Sutures in Body Surgery. Aesthetic. Surg. J. Sep., 1; 33 (3 Supplement): 57S-71S, 2013.

7- Salzberg C.A.: Barbed Sutures in Breast Reconstruction. Aesthetic. Surg. J. Sep., 1; 33 (3 Suppl): 40S-43S, 2013.

8- Karnes J., Morrison W., Salisbury M., Schaeferle M., Beckham P. and Ersek R.A.: Simultaneous breast augmentation and lift. Aesthetic. Plast. Surg., 24 (2): 148-54, 2000.

9- Stevens W.G., Macias L.H., Spring M., Stoker D.A., Chacón C.O. and Eberlin S.A.: One-Stage Augmentation Mastopexy: A Review of 1192 Simultaneous Breast Augmentation and Mastopexy Procedures in 615 Consecutive Patients. Aesthetic. Surg. J. Jul., 1; 34 (5): 723-32, 2014.

10- Spear S.L., Pelletiere C.V. and Menon N.: One-Stage Augmentation Combined with Mastopexy: Aesthetic Results and Patient Satisfaction. Aesthetic. Plast. Surg. Oct., 5; 28 (5): 259-67, 2004.

11- Dancey A., Nassimizadeh A. and Levick P.: Capsular contracture - What are the risk factors? A 14 year series of 1400 consecutive augmentations. J. Plast. Reconstr. Aesthetic. Surg. Feb., 65 (2): 213-8, 2012.

12- Stevens W., Stoker D., Freeman M., Qardt S., Hirsche E. and Cohen R.: Is one-stage breast augmentation with mastopexy safe and effective? a review of 186 primary cases. Aesthetic. Surg. J. Nov., 26 (6): 674-81, 2006.

13- Cannon C.L. and Lindsey J.T.: Conservative augmentation with periareolar mastopexy reduces complications and treats a variety of breast types: A 5-year retrospective review of 100 consecutive patients. Ann. Plast. Surg. May, 64 (5): 516-21, 2010. 